\title{
Vertex potentials evoked during auditory signal detection: Relation to decision criteria*
}

\author{
KENNETH C. SQUIRES \\ Department of Psychology, University of California, San Diego, La Jolla, California 92037 \\ STEVEN A. HILLYARD \\ Department of Neurosciences, University of California, San Diego, La Jolla, California 92037 \\ and \\ PETER H. LINDSAY \\ Department of Psychology, University of California, San Diego, La Jolla, California 92037
}

\begin{abstract}
Vertex potentials were recorded from eight Ss performing in an auditory threshold detection task with rating scale responses. The amplitudes and latencies of both the $\mathrm{N} 1$ and the late positive (P3) components were found to vary systematically with the criterion level of the decision. These changes in the waveshape of the $\mathrm{N} 1$ component were comparable to those produced by varying the signal intensity in a passive condition, but the late positive component in the active task was not similarly related to the passively evoked P2 component. It was suggested that the N1 and P3 components represent distinctive aspects of the decision process, with $\mathrm{N} 1$ signifying the quantity of signal information received and $\mathrm{P} 3$ reflecting the certainty of the decision based upon that information.
\end{abstract}

Mast and Watson (1968) observed that vertex potentials evoked by near threshold auditory stimuli were markedly enlarged and prolonged when Ss actively listened for the signals. This augmentation of evoked activity with attentiveness was most pronounced in a positive wave, which extended from 200 to $450 \mathrm{msec}$ poststimulus. This late positive wave is most likely a composite of a long-latency P2 component (Davis, Mast, Yoshie, \& Zerlin, 1966) of the vertex potential evoked by the low-intensity signal and a P3 or P300 wave associated with the decision (Hillyard, 1969; Smith, Donchin, Cohen, \& Starr, 1970) that task relevant information has been delivered (Sutton, Tueting, Zubin, \& John, 1967).

In order to relate the late positive component to the probabilistic nature of detection near threshold, Hillyard, Squires, Bauer, and Lindsay (1971) averaged evoked potentials separately for each of the four outcomes in a yes-no signal detection task. The P3 wave was evoked only by correctly detected signals (HITS) and not by MISSES, false alarms (FA), or correct rejections (CR). The size of the $\mathrm{P} 3$ wave was found to grow with increasing detectability of the signal, and was larger when a higher criterion for reporting the presence of a signal was adopted. It was concluded that the amplitude of $\mathrm{P} 3$ reflected the level of confidence in the detection of a signal.

In a further analysis of the P3 during signal detection, Paul and Sutton (1972) systematically manipulated the decision criterion by varying the a priori probability of signal presentation and by adjusting the payoff matrix. In both cases, there was a positive correlation between

*This research was supported by NIH Grant NS 07454 and NASA Grant NGR-05-009-83. We thank Donald Norman for providing the experimental facilities.
P3 amplitude and the strictness of the decision criterion. This is consistent with the proposal that $\mathrm{P} 3$ is governed by the confidence of a detection, since those signals which elicit "sensory magnitudes" (Green \& Swets, 1966) great enough to exceed an elevated criterion level are surely detected with greater certainty than those that exceed a less strict criterion.

In some of the tracings shown by Mast and Watson (1968) and Hillyard et al (1971), a negative wave is discernible preceding P3 which also covaries with attentive state and perceptual report. This wave (latency, $100-170 \mathrm{msec}$ ) most likely corresponds to the prominant N1 component of the auditory vertex potential (Davis et al, 1966), which has been related to stimulus intensity (Rapin, Schimmel, Tourk, Krasnegor, \& Pollak, 1966) and to psychophysical judgments of loudness (Keidel \& Spreng, 1965; Davis \& Zerlin, 1966; Davis, Bowers, \& Hirsh, 1968).

Tanis (1972) has recently reported that the amplitude of the N1-P2 complex was correlated with psychophysical judgments in an auditory intensity discrimination task ( 4 vs $5.8 \mathrm{~dB} \quad \mathrm{HL}$ ). The higher intensity signals which were judged high evoked the largest N1-P2, followed by low-intensity signals judged high, high-intensity signals judged low, and finally. low-intensity signals judged low. However, when the evoked potentials were further segregated according to confidence rating responses, no relationship was seen between the N1-P2 amplitude and criterion level.

In the present study, vertex potentials evoked during a threshold-level detection task were averaged according to the S's confidence rating (on an 8-point scale) for detecting the signal, rather than according to a simple yes-no response as in previous studies. In this way. spontaneous trial-to-trial variations of the evoked 
Table 1

Distribution of Trial Types and Derived Signal Detection Parameters as a Function of Confidence Rating (Averaged Across All Eight Ss)

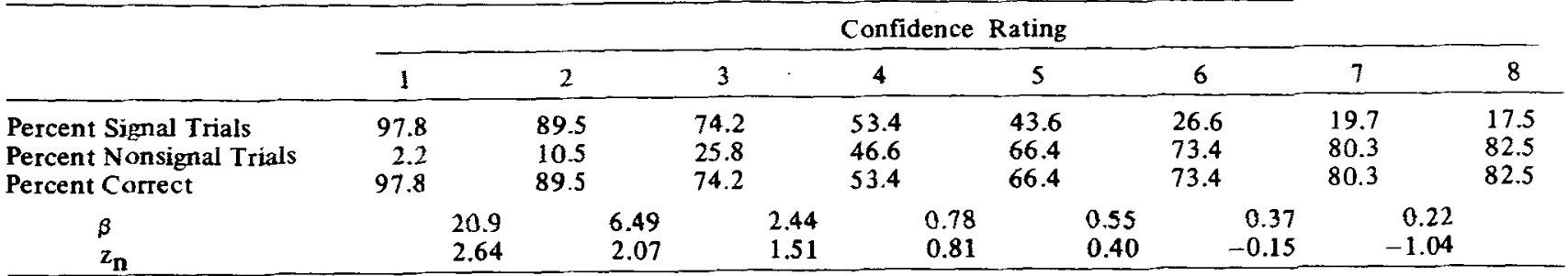

potential could, for the first time, be related to continuous, comparabiy graded measures of perception (Sutton, 1969). The rating scale responses not only provided a direct measure of decision confidence, but also defined a series of decision criteria which were systematically related to both the $\mathrm{N} 1$ and $\mathrm{P3}$ components. It was thereby possible to directly substantiate the "decision certainty" hypothesis regarding the P3 component (Hillyard et al, 1971; Paul \& Sutton, 1972; Squires et al, 1973) and to clarify the relation between the $\mathrm{N} 1$ component and gradations of perceptual experience near threshold.

\section{METHODS}

The Ss were eight normal adults, including Es K.S. and S.H. During testing, the $S$ sat in an acoustically shielded chamber wearing TDH-39 earphones and fixating on a small neon bulb. The task was to decide on each trial whether or not a binaural $1,000-\mathrm{Hz}$ sinusoidal signal of $50 \mathrm{msec}$ duration was present against a background of wide-band white noise (70 dB SPL) continuously present throughout the experiment and to rate his confidence in that decision. A fixed-intensity signal close to detection threshold (defined as $75 \%$ correct in a yes-no procedure), to be used throughout the experiment, was chosen for each $S$.

Each trial began with a 200-msec flash of the fixation bulb which served as a warning signal. On one-half of the trials, selected at random, the offset of the warning signal was followed after $500 \mathrm{msec}$ by the $1,000-\mathrm{Hz}$ signal; on the other trials, no signal was presented. No additional stimulus served to mark the point at which the signal might occur. The neon bulb was relit $2.0 \mathrm{sec}$ after the warning light, thereby directing the $S$ to respond by pressing one of eight numbered buttons on the panel before him. When highly confident that a signal had been presented, he pressed Button 1; Ratings 2, 3, and 4 indicated decreasing confidence in signal presence; a 5 indicated marginal confidence that the trial had been a nonsignal trial; and 6,7 , and 8 indicated increasing confidence that there had not been a signal. A feedback light was provided $1 \mathrm{sec}$ after each buttonpress to inform the $S$ whether or not a signal had been present. Intertrial intervals were randomized between 4 and 6 sec.

Each $S$ was given enough experience in the task to stabilize his distribution of judgments along the rating scale before evoked potential recording was begun. Trials were presented in blocks of 70 , with four or five blocks per 2 -h testing session. Each $S$ participated in from three to seven recording sessions.

Evoked potentials were recorded from the vertex referred to the right mastoid using $\mathrm{Ag}-\mathrm{AgCl}$ electrodes (Beckman) and amplified with Grass P-15 amplifiers (bandpass $0.1-300 \mathrm{~Hz}$ ). Evoked potentials were computer-averaged separately according to the 16 outcomes formed by the 8 numerical response categories and 2 signal conditions. Averaging epochs were either 500 or $750 \mathrm{msec}$ in duration, beginning $500 \mathrm{msec}$ after the warning light offset. Additional recordings were taken in a "passive" condition, wherein the $S$ read a book while the signals were presented at intervals identical to those used in the active task. No trial lights were visible to the $S$ during this condition.

The vertical electro-oculogram was either monitored on-line or averaged to ensure absence of eye-movement artifacts.

Stimulus timing, signal presentation, and evoked response averaging were under the control of a PDP-9 computer. Measurements of evoked potential parameters were also performed with this computer.

\section{RESULTS}

\section{Detection Performance}

All Ss readily learned to categorize their perceptual judgments according to the 8-point confidence rating scale. Their distributions of responses were stable over blocks of trials and across testing sessions. The first two rows of Table 1 indicate the validity of the psychophysical judgments. Small numerical ratings were associated with a high percentage of signal trials and the higher numerical ratings associated with a high percentage of nonsignal trials. Accordingly, the percentage of correct decisions increased systematically from the central, low-confidence ratings out to either extreme (Row 3, Table 1).

Quantitative measures of the decision criterion for each confidence level can be calculated from such rating scale data (see Green \& Swets, 1966). According to signal detection theory, the two signal conditions (signal present and noise alone) elicit "sensory events" in the nervous system which vary stochastically in magnitude from one presentation to the next. The confidence level of a decision regarding signal presence or absence is determined by comparing the sensory magnitude observed on each trial with a set of criterion levels. In this case, seven criterion cutoffs separate the eight response categories. In the last two rows of Table 1 , the seven criterion values are expressed in two alternative forms: in units of standard deviation $\left(z_{n}\right)$ above the mean of the hypothetical underlying distribution of sensory events resulting from noise alone, and as likelihood ratios $(\beta)$. (The likelihood ratio is the ratio of the probabilities that a given sensory event resulted from a signal presentation and from noise alone). For these 
calculations, normal distributions of sensory magnitude with unequal variance were assumed (see Green \& Swets, 1966, pp. 94-96). It is evident from Table 1 that the confidence rating technique effectively established a wide range of criterion levels over which the perceptual reports were distributed.

\section{Evoked Potentials and Perceptual Reports}

The 16 average evoked potential waveforms for E.K. are shown in Fig. 1. For the highest confidence HIT (a rating of 1 , signal present), there was a large negative component (N1), with a peak latency of $165 \mathrm{msec}$ followed by a broad positive component peaking at $365 \mathrm{msec}$. This positive peak will be termed P3, although it is probably composed in part of the $\mathrm{P} 2$ component of the vertex potential. For progressively less confident decisions, these components diminished in amplitude and increased in latency until Level 4 , where they were generally indistinguishable from waveform noise. No evoked components were distinguishable in the waveforms for the other three general categories (MISSES, FAs, and CRs). Also included in Fig. 1 are the combined waveforms across all rating levels.

Similar results are seen for K.S. in Fig. 2 and N.H. in

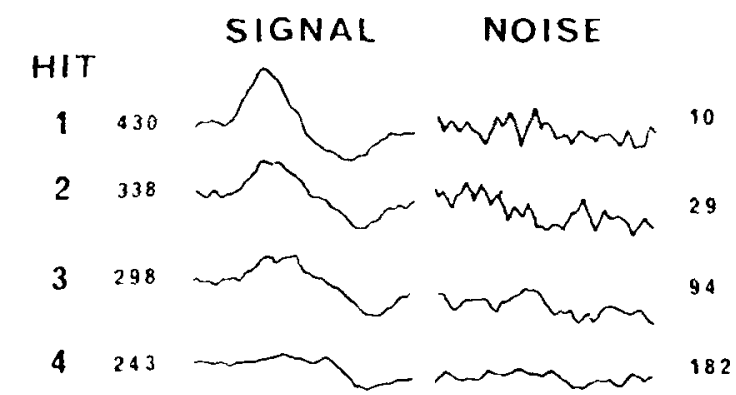

MISS

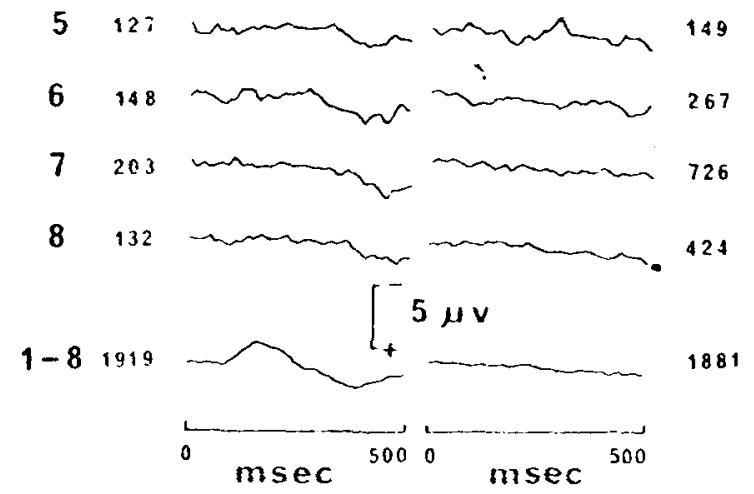

Fig. 1. Vertex potentials evoked by each combination of confidence rating and signal condition for E.K. Signal onset is at time zero in left column. Numbers 1.8 at left indicate the confidence rating response. Subheadings indicate the four general categories of outcome. Bottom traces show the overall average waveform across all eight ratings. Number beside each trace indicates the number of trials included in each average. Polarity convention, vertex positivity downward.

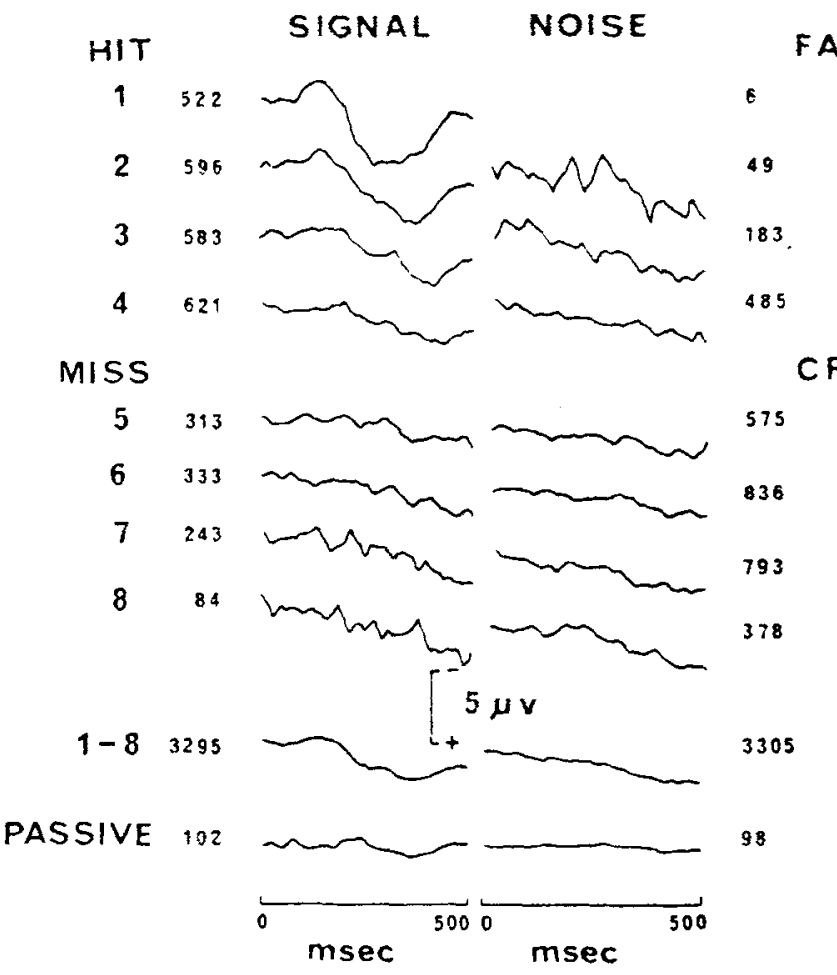

Fig. 2. Same as Fig. 1 for K.S. Also included is the vertex potential evoked by the signal used in the active task during the passive (reading) condition. The waveform for the highest confidence $F A$ is omitted due to an insufficient number of trials.

Fig. 3 (note the change of time base to $750 \mathrm{msec}$ for N.H.). In Figs. 2 and 3 , the potentials evoked by the signals used in the active task but recorded in the passive condition are also shown. Equivalent waveforms were obtained for the other five Ss.

In Fig. 4, the mean amplitude of the $\mathrm{N} 1$ and $\mathrm{P} 3$ components for all $\mathrm{Ss}$ (expressed as percentages of the maximum value for each $S$ ) are presented as a function of confidence rating. The amplitudes of $\mathrm{N} 1$ and $\mathrm{P} 3$ were taken as the largest negative and positive deflections between 100 and $200 \mathrm{msec}$ and between 250 and $450 \mathrm{msec}$, respectively, referred to a baseline of the average potential over the first $60 \mathrm{msec}$ of the recording interval. All Ss showed a systematic decrease in both N1 and P3 amplitudes as the confidence level for HITS decreased $[\mathrm{F}(3,21)=17.46, \mathrm{p}<.001$, for $\mathrm{N} 1 ; \mathrm{F}(3,21)=$ $8.87, p<.001$, for $\mathrm{P} 3]$. Since the $\mathrm{N} 1$ and P3 components for the lowest confidence HITS were not always discernible, and since none could be determined for any level of MISS, FA, or CR, these points are indicative of the noise level of the averaged wavefoms.

Highly confident FAs and MISSES occurred rarely. and any evoked activity associated with these decisions would be less precisely time-locked to the averaging epoch, making for relatively "noisy" and indistinct waveforms. To obtain better signal-to-noise ratios for the P3 component. the eight evoked waveforms for each signal condition were collapsed into four. representing 


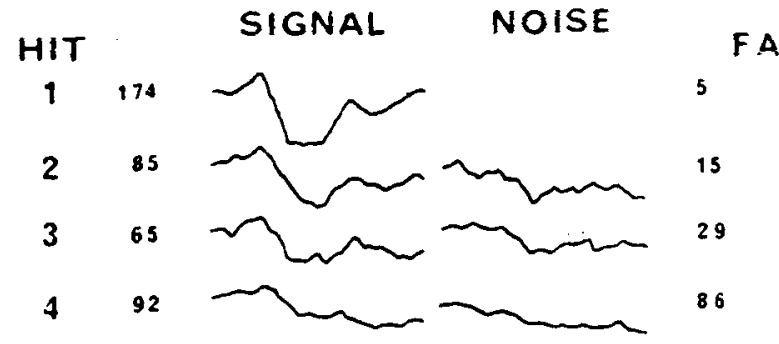

MISS

C R

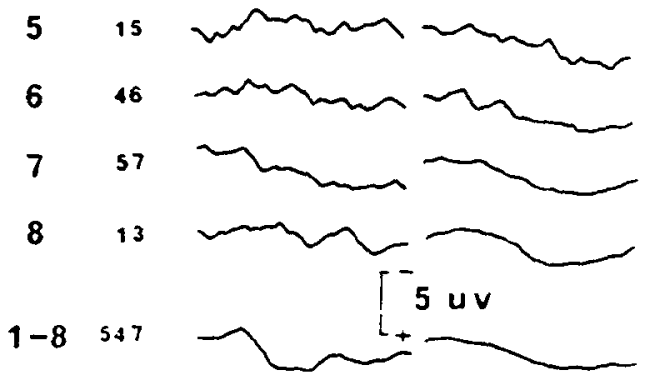

25

73

240

170

643

PASSIVE 100

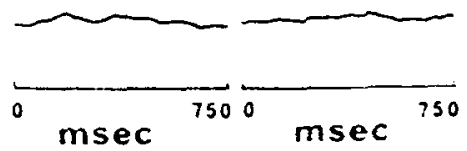

100

Fig. 3. Same as Fig. 2 for N.H.

high- and low-confidence signal-present waveforms (Levels 1-2 and 3-4) and high- and low-confidence noise-alone waveforms (Levels 7-8 and 5-6, respectively). The $\mathrm{P} 3$ area was calculated for each collapsed waveform within the latency range of $300-450 \mathrm{msec}$, referred to the mean voltage "baseline" of the first $60 \mathrm{msec}$ of the averaging epoch (Fig. 5); for signal-present trials (HITS and MISSES), the relationship between the P3 area and confidence rating was highly significant $[F(3,21)=$ $24.13, p<.001]$. Specific comparisons showed high-confidence HITS, had larger $\mathrm{P} 3$ areas than low $[F(1,21)=8.53, p<.01]$, while low-confidence HITS, low-confidence MISSES, and high-confidence MISSES did not differ significantly. Similarly, for noise-only trials (FAs and CRs), the overall relationship between P3 area and rating was highly significant $[F(3,21)=8.66$, $\mathrm{p}<.001$ ] . High-confidence FAs had larger P3 areas than did low-confidence $F A s[F(1,21)=16.06, p<.001]$, which did not differ significantly from those on low-confidence CRs and high-confidence CRs. The P3 area for high-confidence HITS was marginally larger than for high-confidence $F A s[F(1,7)=4.15, p<.10]$.

The latencies of the positive and negative peaks are plotted in Fig. 6 as a function of confidence level for HITS. The data for N.S. were omitted in Fig. 6 because an N1 component could not be determined for Level 3 . The average shift in latency was $32 \mathrm{msec}$ for N1 across Levels $1-3[F(2,12)=10.06, p<.005]$ and $85 \mathrm{msec}$ for $P 3$ across Levels $1.4[F(3,21)=16.62, p<.001] .^{1}$

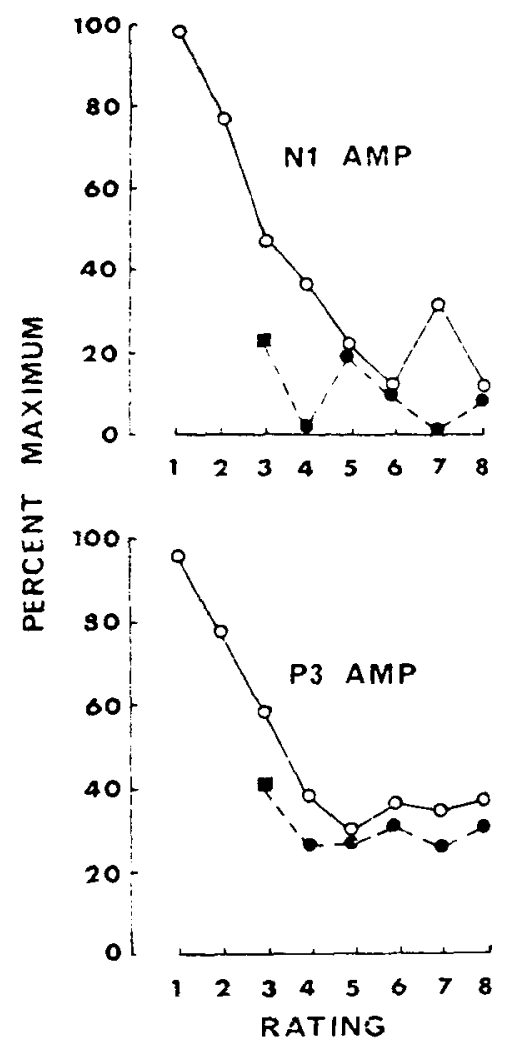

Fig. 4. Amplitude of $\mathrm{N} 1$ and $\mathrm{P} 3$ as a function of confidence rating, relative to a baseline of the average voltage over the first $60 \mathrm{msec}$ of the recording interval, and expressed as the percentage of the maximum value for each $S$. Averaged over all eight $\mathrm{Ss}$, except for the square symbols which include six Ss for $\mathrm{N} 1$ and seven Ss for P3. Open circles are for trials with signal present and closed circles are for signal-absent trials.

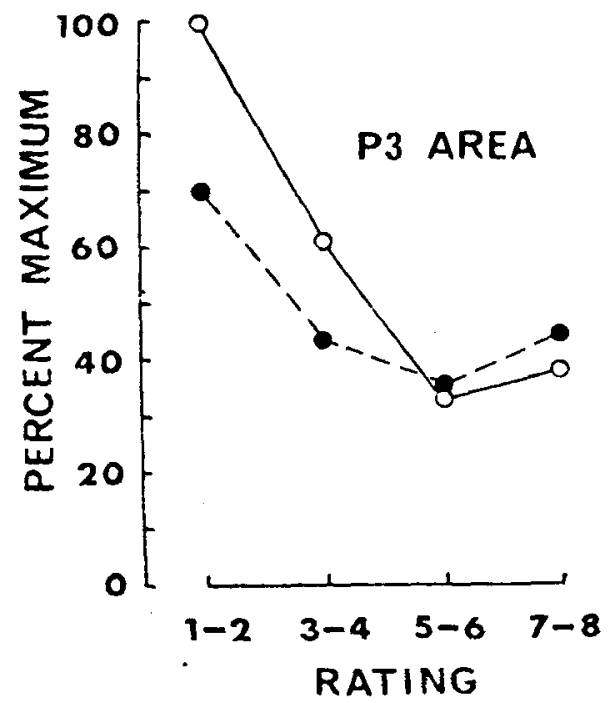

Fig. 5. Area of $P 3$ between 300 and $450 \mathrm{msec}$ as a function of confidence rating, relative to a baseline of the average voltage over the first $60 \mathrm{msec}$ of the recording interval, and expressed as the percentage of the maximum value for each $S$. The original eight rating categories have been collapsed into four. Average of eight Ss. Open symbols for signal present and closed for noise alone. 


\section{Comparison of Active and Passive Evoked Potentials}

It is well known (e.g., Rapin et al, 1966) that the N1 evoked by tone burst signals increases in latency and decreases in amplitude as the signal intensity is reduced to low levels. The question arose as to whether the reciprocal amplitude-latency relation seen with decreasing confidence rating was simply an effect of a reduction in effective stimulus intensity or "loudness" of the signal. To evaluate this possibility, additional evoked potentials from four Ss (N.S., N.H., K.S., and T.P.) were recorded in a passive condition (reading a book) for each of the five to seven signal intensities, which ranged from the one used in the active task up to 15-25 dB more intense.

The inverse function relating the amplitude and latency of N1 evoked under passive conditions is plotted in Fig. 7 for each S (open circles). The function relating the amplitude and latency of $\mathrm{N} 1$ as the confidence level declines (closed circles) is superimposable upon the function described by the passively evoked N1 as intensity varies. In other words, the $\mathrm{N} 1$ component varies with decreasing confidence rating as if the signal intensity had been reduced. The Nl amplitude and latency for the highest confidence HIT were equivalent to those for the passive $\mathrm{N} 1$ elicited by a signal $8 \mathrm{~dB}$ more intense than the one used in the active task.

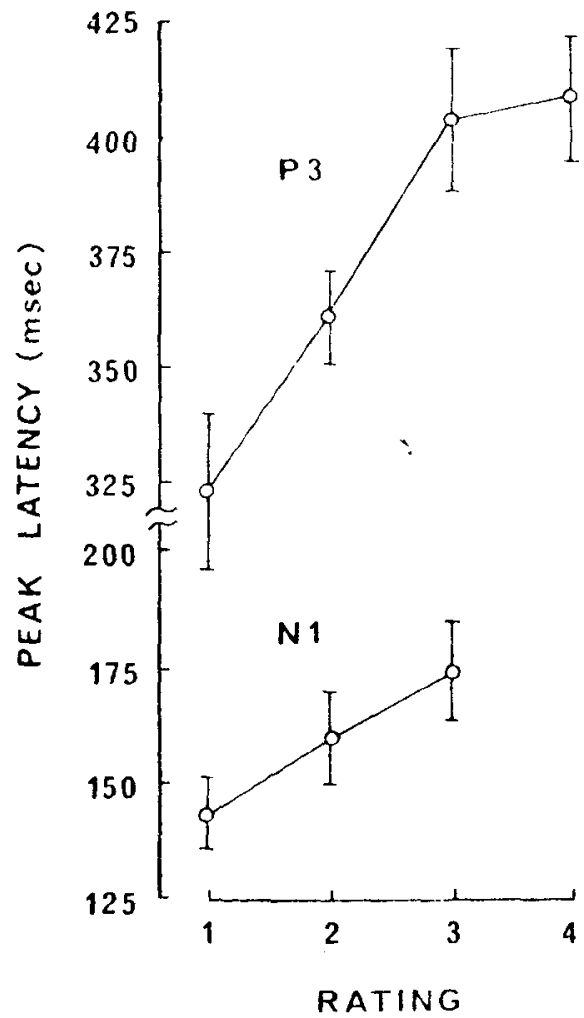

Fig. 6. Latencies of $\mathrm{N} 1$ and P3 peaks on HIT trials. Plotted as a function of confidence rating. Average of seven Ss. Brackets indicate one standard error.

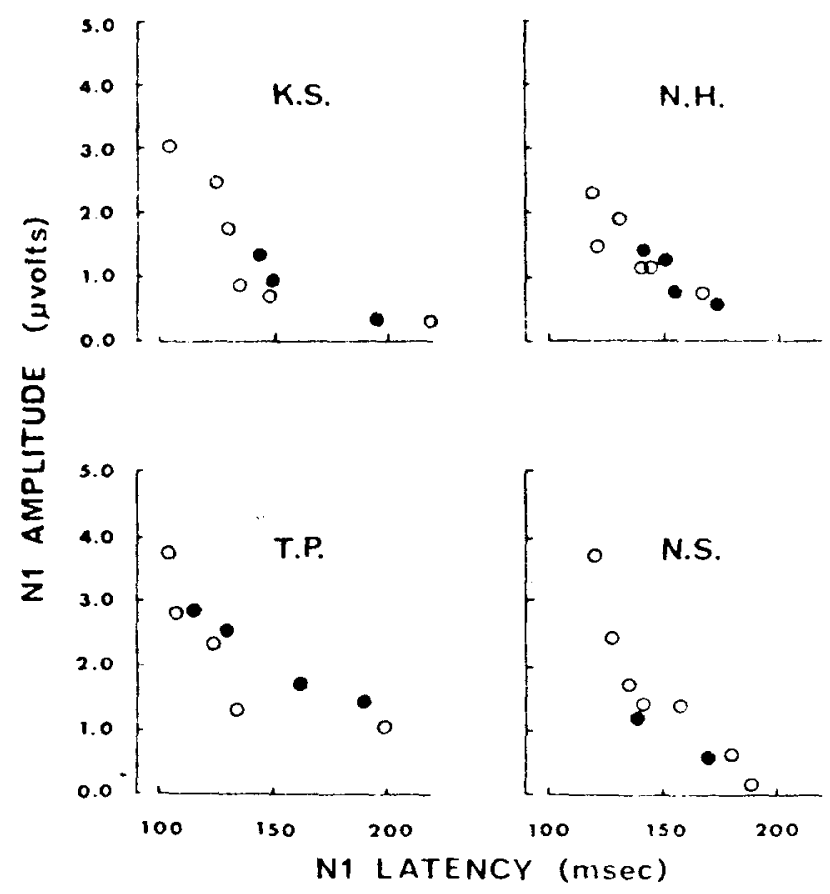

Fig. 7. Plots of N1 amplitude vs latency for four Ss. Open circles represent values from the passive condition as signal intensity is varied and closed circles represent values from the active condition sorted according to confidence rating.

For the large positive component which follows N1, however, there is a striking difference in the amplitude-latency functions between active and passive conditions. This relationship is shown in Fig. 8 (circles). The amplitude of the positive component associated with the active task is considerably greater for a given latency than that of the P2 component elicited in the passive condition. In some waveforms for N.S., T.P., and K.S., an inflection was discernible on the positive-going wave, with a latency between 200 and $250 \mathrm{msec}$. The amplitude-latency plots of these points (triangles) fall reasonably close to those of the $\mathrm{P} 2$ peaks in the passive condition.

\section{DISCUSSION}

By computer averaging vertex potentials evoked by very faint tone bursts into eight separate categories according to the S's rated confidence that he had heard the signal, a marked trial-to-trial variability of evoked waveform was revealed. Both the N1 (latency, $150-200 \mathrm{msec}$ ) and the late positive component (latency, $300-450 \mathrm{msec}$ ) systematically increased in amplitude and decreased in latency as a function of the confidence of correct signal detections. No evoked potential components were discernible following signals that went undetected at any level of certainty. In previous studies (Hillyard et al, 1971: Paul \& Sutton, 1972), wherein Ss were allowed only two categories of perceptual report (signal present or signal absent). it was unavoidable that individual evoked potentials having very difterent 

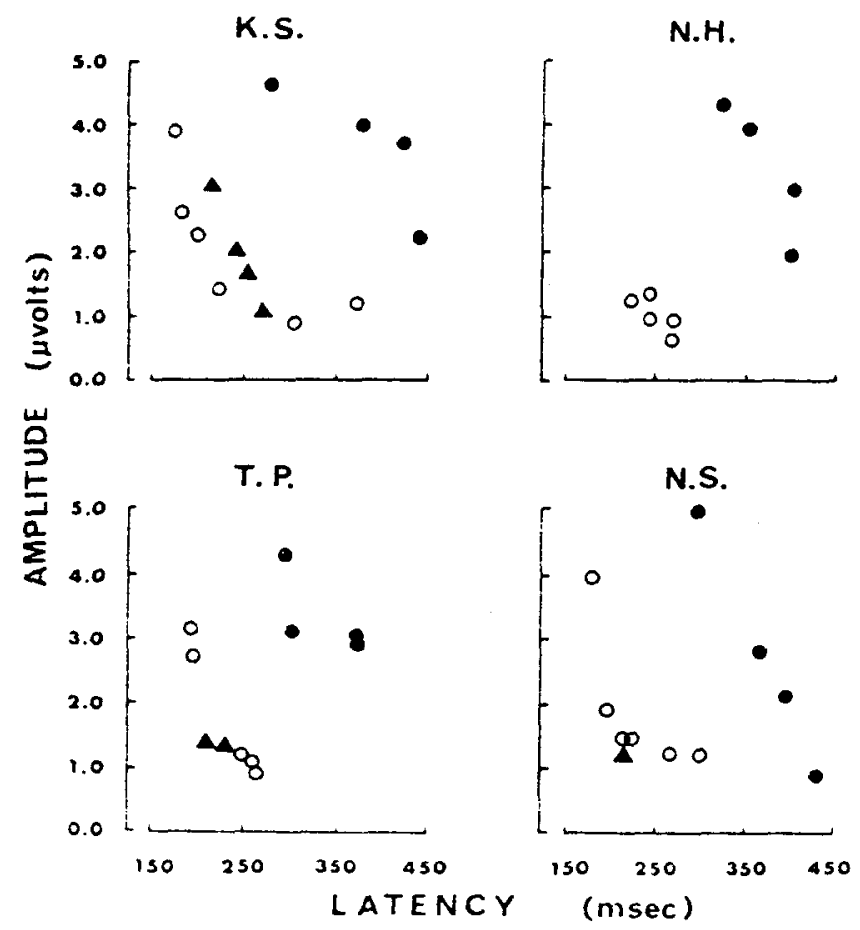

Fig. 8. Amplitude-latency functions for the major positive deflections for four $S$ s in the passive condition (open circles) and active condition (closed circles), and for the inflection point (closed triangles) on the positive-going limb of the late wave that was observed in some of the waveforms for the active condition.

waveforms were averaged together in the overall average for HITS. The failure of these previous studies to observe a distinct, sharp N1 component on HIT trials is probably due to its "washing out" in the averaging process because of its variable latency. Such an averaged waveform not only conceals the enormous variability of threshold-level evoked potentials, but may not, in fact, represent any actual evoked potential. As Sutton (1969) has pointed out, evoked potentials are continuously graded phenomena, and to reveal their actual relationship to perception, it is necessary to segregate them according to comparably graded perceptual categories.

Increasingly confident decisions were correct a higher percentage of the time, indicating that the confidence ratings were based upon signal information rather than randomness in the decision making. Following signal detection theory, higher confidence ratings are equivalent to decisions made with a higher criterion cutoff (Green \& Swets, 1966). Our paradigm generated very strict criteria for the most confident signal-present responses (Table 1). Since the N1 component was largest and most distinct on the highest criterion HITS, the absence of a clear N1-criterion relationship in the data of Paul and Sutton, and the lack of a relation between N1-P2 and confidence rating reported by Tanis (1972) may have been due to the narrower range of criterion values produced by their manipulations. The close relationship of $\mathrm{N} 1$ amplitude to the criterion for detections suggests that N1 may serve as an electrophysiological correlate of the "sensory magnitude" that is presumed to underlie the S's decision (Green \& Swets, 1966). The report by Tanis that N1-P2 in a two-choice intensity discrimination decrease progressively in amplitude in the order of HIT, FA, MISS, and CR judgments (where a high-intensity signal correctly identified is termed a HIT, etc.) is consistent with this proposal.

The strong dependence of the $\mathrm{N} 1$ and late positive components upon decision criterion must be taken into account when examining the question of how evoked potential and behavioral thresholds are related (Sutton, 1969; Donchin \& Sutton, 1970). Our results indicate that repeated presentations of any fixed "threshold" level signal will generate a continuously graded set of evoked potential waveforms which parallel the perceptual continuum of decision confidence. This is consistent with the model underlying signal detection theory, which assumes a continuum of sensory magnitudes rather than a dichotomy of perception above and below threshold. Accordingly, the amplitude of an averaged evoked potential to such signals will depend upon how the trials are selected for averaging in relation to the decision criterion.

A number of sources might contribute to the trial-to-trial covariability of evoked potential waveform and confidence rating. Peripheral acoustic variations could arise from (1) the stochastic nature of the background noise, (2) breathing sounds, (3) noise of aural vascular pulsations, or (4) spontaneous middle-ear muscle activity. A certain amount of "neural noise" results from spontaneous firing of neurons in the auditory pathway. Finally, fluctuations of central states such as arousal, alertness, or attentive strategy might alter these evoked potential components.

Some of the differences in averaged evoked potential amplitude among the rating categories might have resulted from differences in the variability of peak latencies of the components within the categories, which was not ascertained. Similarly, the comparison of evoked potential amplitudes on passive trials with those during active detection is confounded with possible differences in the variability of peak latencies within those conditions. However, the evoked response averaged over all active trials was substantially larger than the passively recorded evoked potential to the same signal (Figs. 2 and 3 ), which suggests a net increase in evoked potential amplitude due to attentiveness, since it is unlikely that the variability on the passive run would exceed the marked variability shown for the active condition. Assuming that the evoked components within a rating category have approximately the same latency variance as do the passively recorded evoked responses at a somewhat higher signal intensity, we estimate that the $\mathrm{N} 1$ wave for the most confident rating has the same amplitude and latency as a passively recorded potential 
elicited by a signal that is about $8 \mathrm{~dB}$ more intense.

The comparison of evoked potentials on active and passive conditions suggests that the $\mathrm{N} 1$ and late positive components, while being highly correlated with each other and with performance measures, are related to the sensory processing in fundamentally different ways. The similarity of the amplitude vs latency plots of N1 with confidence rating to that determined by changing the stimulus intensity suggests that $\mathrm{N} 1$ variability in detection results from trial-to-trial changes in effective stimulus intensity. However, the possibility remains that a central factor, such as attentiveness, might act to increase $\mathrm{N} 1$ amplitude on active trials without causing a proportionate shortening of its latency, as the waveforms shown by Mast and Watson (1968) and Tanis (1972) would suggest. A final consideration is that the delayed $\mathrm{N} 1$ wave might represent, in part, the negative component of the "decision complex" that includes the P3 (Picton, Hillyard, \& Galambos, in press).

An additional line of evidence that the N1 variations with rated confidence are equivalent to changing signal strengths comes from the psychophysical data of Green, McKey, and Licklider (1959). They found that the psychometric functions for detecting auditory signals spanned some 7 to $11 \mathrm{~dB}$ between chance and perfect performance. Similarly, we found that the growth of N1 across Ratings 1 through 4, which yielded from near chance to near perfect performance, was equivalent to that produced by incrementing the signal by $8 \mathrm{~dB}$ in the passive condition. In other words, if a signal evokes a larger N1, either because it is physically more intense or because of spontaneous variability in the processing sequence, that signal will be distinguishable from the background noise with a correspondingly higher probability. This indicates that $\mathrm{N} 1$ evoked by low-level signals indexes an aspect of the sensory input that is utilized in making the detection judgment, and possibly reflects such perceptual qualities as "sensory magnitude" or the "distinctiveness" of the signal in noise.

The prominent late positive component observed on HIT trials did not follow the same amplitude-latency function as the passively evoked $\mathbf{P} 2$ component, but was relatively much greater in amplitude for a given latency. The major contributor to this late positivity would therefore seem to be the wave variously known as P3, $\mathrm{P} 300$, decision complex, or association cortex potential (Sutton, 1969; Hillyard, 1969; Smith et al, 1970; Picton et al, in press; Ritter, Simson, \& Vaughan, 1972), which reflects the S's decision regarding the signal. In some Ss, an inflection was discernible on the positive-going limb of the late positive wave which did, in fact, superimpose upon the same amplitude-latency function as the passively recorded $\mathrm{P} 2$. The entire late positive wave therefore seems to be composed of a small, delayed P2 and a large P3. A further distinction between the P2 and P3 components derives from scalp distribution studies (Vaughan, 1969); the maximum-amplitude P2 wave is recorded anteriorly on the scalp relative to that for the
P3, as is evident in the tracings of Kostandor and D'yachkova (1972) recorded during threshold detection.

The growth of P3 on HIT trials with higher confidence rating is consistent with the hypothesis that this wave is an index of the certainty of an affirmative decision (Hillyard, 1969; Hillyard et al, 1971; Squires et al, 1973). This hypothesis predicts a positive relation between P3 amplitude on HITS and decision criterion, since as criterion is raised HIT decisions are based upon improved sensory evidence and thus are more confident. This has been verified for criterion levels derived from confidence ratings and by varying the signal probability or payoff matrix (Paul \& Sutton, 1972). Paul and Sutton also suggested that a raised criterion might make a signal more "salient," which in turn would enhance the P3. Another consequence of a criterion elevation is that signals falling into the more restricted HIT category are more improbable and may evoke a larger P3 on that basis (Sutton, 1969; Tueting, Sutton, \& Zubin, 1971).

The area of P3 on highly confident FA trials was greater than that associated with CRs and MISSES, but did not differ markedly from that accompanying HIT decisions. This indicates that P3 is associated with "detect" decisions in proportion to confidence rating, regardless of the correctness of the decision, as specified by the "confidence hypothesis." The absence of a P3 on FA trials reported in prior yes-no experiments (Sutton, 1970; Hillyard et al, 1971) can most likely be attributed to the low confidence level of those decisions. The present study reveals that highly confident FAs are seldom made, so that a combined average over all FA would be dominated by the more numerous low-confidence decisions. In general, however, for a given confidence level, the averaged P3 for a HIT is larger and more distinct than for a FA. This might be attributed to two factors: first, according to signal detection theory, the FA decisions at each confidence rating would be distributed more toward the lower criterion cutoff than are HITS, and second, in the absence of an external signal "marker" other than the warning light, FA decisions will be less precisely time-locked to the computer-averaging epoch than HITS.

The absence of a P3 component on CR trials (Hillyard et al, 1971; Paul \& Sutton, 1972) was verified, even for the most confident ratings. This finding militates against the idea that P3 is determined by the certainty of any type of decision or the delivery of any type of information; a HIT of Level 2 resolves as much uncertainty as a CR of Level 8 (equal percent correct), yet the former has a much larger P3. This might be explained by assuming that the S's attentive strategy is to match sensory input only against an internal "template" or "model" of the signal (Squires et al, 1973), and not against an indeterminate "noise" template. In other types of sensory decision tasks, however, where two signal alternatives are equally distinctive, P3 components may be evoked by either one (Sutton et al, 1967: Tueting et al, 1971; Karlin. Martz, 
Brauth, \& Mordkoff, 1971; Squires et al, 1973; Posner, Klein, Summers, \& Buggie, 1973), suggesting that input was matched against both templates.

The close relationship among N1, P3, and decision criterion is consistent with the hypothesis that $\mathrm{N} 1$ reflects the amount of signal information that is utilized in the decision process and $\mathrm{P} 3$ represents the certainty of the decision based upon that information. Since the small long-latency $\mathrm{N} 1$ associated with low-confidence ratings delivers less distinct information than a larger one, the subsequent P3 should be even more delayed in latency since it would take longer to process the indistinct information and arrive at a decision. Indeed, N1 latency was found to increase by $32 \mathrm{msec}$ between Levels 1 and 3, while P3 latency increased by more than 80 msec. Ritter et al (1972) have obtained direct evidence that P3 latency is related to decision latency in a difficult discrimination-reaction time task. They also estimated that the onset of P3 occurs early enough to be coincident with the actual decision-making process, but that most of the positivity is subsequent to the decision. This suggests that the P3 component might represent the sequelae of the decision, such as its emotional significance or the activation of the action program or appropriate response set (cf. Karlin \& Martz, 1972).

\section{REFERENCES}

Davis, H., Bowers, C., \& Hirsh, S. Relations of the human vertex potential to acoustic input: Loudness and masking. Journal of the Acoustical Society of America, 1968, 43, 431-438.

Davis, H., \& Zerlin, S. Acoustic relations of the human vertex potential. Journal of the Acoustical Society of America, 1966, 39, 109-116.

Davis, H., Mast, T., Yoshie, N., \& Zerlin, S. The slow response of the human cortex to auditory stimuli: Recovery process. Electroencephalography \& Clinical Neurophysiology, 1966, 21, 105-113.

Donchin, E., \& Sutton, S. The "psychological significance" of evoked responses: A comment on Clark, Butler and Rosner. Communications in Behavioral Biology, 1970, 5, 111-114.

Green, D., \& Swets, J. Signal detection theory and psychophysics. New York: Wiley, 1966.

Green, D., McKey, M., \& Licklider, J. Detection of a puised sinusoid in noise as a function of frequency. Journal of the Acoustical Society of America, 1959, 31, 1446-1452.

Hillyard, S. A. The CNV and the vertex evoked potential during signal detection: A preliminary report. In $\mathrm{E}$. Donchin and D. B. Lindsley (Eds.), Average evoked potentials: Methods, results, and evaluations. Washington, D.C: National Aeronautics \& Space Administration, 1969. Pp. 349-354.

Hillyard, S., Squires, K., Bauer, J., \& Lindsay, P. Evoked potential correlates of auditory signal detection. Science, $1971,172,1357-1360$.

Karlin, L., Martz, M., Brauth, S., \& Mordkoff, A. Auditory evoked potentials, motor potentials, and reaction time. Electroencephalography \& Clinical Neurophysiology, 1971, 31, 129-136.

Karlin, L., \& Martz, M. Response probability and sensory evoked potentials. In S. Kornblum (Ed.), Attention and performance
$I V$. New York: Academic Press, 1972.

Keidel, W., \& Spreng, M. Neurophysiological evidence for the Stevens power function in man. Journal of the Acoustical Society of America, 1965, 38, 191-195.

Kostandov, E. A., \& D'yachkova, G. 1. Evoked potentials of the human cerebral cortex to perceptible and imperceptible sound stimuli. Neirofiziologiya, 1971, 2, 115-122.

Mast, T., \& Watson, C. Attention and auditory evoked responses to low-detectability signals. Perception \& Psychophysics, $1968,4,237-240$.

PauI, D., \& Sutton, S. Evoked potential correlates of response criterion in auditory signal detection. Science, 1972, 177, 362-364.

Picton, T., Hillyard, S., \& Galambos, R. Evoked responses to omitted stimuli. In M. N. Livanov (Ed.), Major problems of brain electrophysiology. Moscow: USSR Academy of Science, in press.

Posner, M. I., Klein, R., Summers, J., \& Buggie, S. On the selection of signals. Memory \& Cognition, 1973, 1, 2-12.

Rapin, I., Schimmel, H., Tourk, L., Krasnegor, N., \& Pollak, C. Evoked responses to clicks and tones of varying intensity in waking adults. Electroencephalography \& Clinical Neurophysiology, 1966, 21, 335-344.

Ritter, W., Simson, R., \& Vaughan, H. G., Jr. Association cortex potentials and reaction time in auditory discrimination. Electroencephalography \& Clinical Neurophysiology, 1972, $33,547-556$.

Smith, D. B. D., Donchin, E., Cohen, L., \& Starr, A. Auditory averaged evoked potentials in man during selective binaural listening. Electroencephalography \& Clinical Neurophysiology, 1970, 28, 146-152.

Squires, K., Hilly ard, S., \& Lindsay, P. Cortical potentials evoked by confirming and disconfirming feedback following an auditory discrimination. Perception \& Psychophysics, 1973, $13,25-31$.

Sutton, $S$. The specification of psychological variables in an average evoked potential experiment. In E. Donchin and D. B. Lindsley (Eds.), Average evoked potentials: Methods, results, and evaluations. Washington, D.C: National Aeronautics \& Space Administration, 1969. Pp. 237-298.

Sutton, $S$. The sensitivity of the evoked potential to psychological variables. Paper presented at the American EEG Society, September 1970, Washington, D.C.

Sutton, S., Tueting, P., Zubin, J., \& John, E. R. Information delivery and the sensory evoked potential. Science, 1967, 155, 1436-1439.

Tanis, D. A signal-detection analysis of auditory evoked potentials in an intensity discrimination task. Doctoral dissertation, Washington University, 1972.

Tueting, P., Sutton, S., \& Zubin, J. Quantitative evoked potential correlates of the probability of events. Psychophysiology, 1971, 7, 385-394.

Vaughan, H. G., Ir. The relationship of brain activity to scalp recordings of event-related potentials. In E. Donchin and D. B. Lindsley (Eds.), Averaged evoked potentials: Methods, results and evaluations. Washington, D.C: National Aeronautics \& Space Administration, 1969. Pp. 45-75.

\section{NOTE}

1. Latency shifts for N.S., omitted in Fig. 6, were $30 \mathrm{msec}$ for $\mathrm{N} 1$ between Levels 1 and 2 and $125 \mathrm{msec}$ for P3 between Levels 1 and 4.

(Received for publication January 11, 1973; revision received March 30, 1973.) 